\title{
Hypercube Subgraphs with Local Detours
}

\author{
Peter Hamburger, ${ }^{1 *}$ Alexandr V. Kostochka, ${ }^{2}$ \\ Alexander Sidorenko ${ }^{3}$ \\ ${ }^{1}$ DEPARTMENT OF MATHEMATICAL SCIENCES \\ INDIANA UNIVERSITY — PURDUE UNIVERSITY FORT WAYNE \\ FORT WAYNE, INDIANA 46805 \\ E-mail: hamburge@indiana.edu \\ 2 INSTITUTE OF MATHEMATICS \\ 630090 NOVOSIBIRSK, RUSSIA \\ E-mail:sasha@math.nsc.ru \\ ${ }^{3}$ COURANT INSTITUTE OF MATHEMATICAL SCIENCES \\ NEW YORK UNIVERSITY \\ NEW YORK, NY 10012 \\ E-mail: sidorenk@cims.nyu.edu
}

Received May 16, 1997; revised August 21, 1998

\begin{abstract}
A minimal detour subgraph of the $n$-dimensional cube is a spanning subgraph $G$ of $Q_{n}$ having the property that, for vertices $x, y$ of $Q_{n}$, distances are related by $d_{G}(x, y) \leq d_{Q_{n}}(x, y)+2$. For a spanning subgraph $G$ of $Q_{n}$ to be a local detour subgraph, we require only that the above inequality be satisfied whenever $x$ and $y$ are adjacent in $Q_{n}$. Let $f(n)$ (respectively, $f_{l}(n)$ ) denote the minimum
\end{abstract}

* Dedicated to the memory of Paul Erdős

Contract grant sponsor: DIMACS. DIMACS is a cooperative project of Rutgers University, Princeton University, AT\&T Labs, Bell Labs and Bellcore. DIMACS is an NSF Science and Technology Center.

Contract grant number: STC-91-19999.

Contract grant sponsor: New Jersey Commission on Science and Technology Contract grant sponsor: Russian Foundation for Fundamental Research. Contract grant numbers: 96-01-01614 and 97-01-01075

(c) 1999 John Wiley \& Sons, Inc.

CCC 0364-9024/98/020101-11 
number of edges in any minimal detour (respectively, local detour) subgraph of $Q_{n}$ (cf. Erdös et al. [1]). In this article, we find the asymptotics of $f_{l}(n)$ by showing that $3 \cdot 2^{n}\left(1-\mathcal{O}\left(n^{-1 / 2}\right)\right)<f_{l}(n)<3 \cdot 2^{n}(1+o(1))$. We also show that $f(n)>$ $3.00001 \cdot 2^{n}$ (for $n>n_{0}$ ), thus eventually $f_{l}(n)<f(n)$, answering a question of [1] in the negative. We find the order of magnitude of $F_{l}(n)$, the minimum possible maximum degree in a local detour subgraph of $Q_{n}: \sqrt{2 n+0.25}-0.5 \leq$ $F_{l}(n) \leq 1.5 \sqrt{2 n}-1$. (c) 1999 John Wiley \& Sons, Inc. J Graph Theory 30: 101-111, 1999

Keywords: hypercube, minimal detour, local detour

\section{INTRODUCTION}

Parameters such as Hamming distances, distance sums, connectedness, maximum degree, diameter, and the size of a minimum dominating set in the hypercube and its spanning subgraphs have been investigated in many articles. Erdös, Hamburger, Pippert, and Weakley [1] introduced and studied a combination of these types of parameters. Some problems, questions, and conjectures were raised.

We say that a subgraph $G$ of the $n$-dimensional hypercube $Q_{n}$ has the $k$-detour property if any two vertices at distance $d \leq k$ in $Q_{n}$ are at distance at most $d+2$ in $G$. Note that if $k=1$, the definition gives the notion of a local detour subgraph, and if $k=n$, it yields the notion of a minimal detour subgraph of [1]. Let $f(n)$ and $f_{l}(n)$ denote the minimum number of edges of any minimal detour subgraph or any local detour subgraph of $Q_{n}$, respectively. Further, let $F_{l}(n)$ denote the minimum possible maximum degree in any local detour subgraph of $Q_{n}$.

Clearly every minimal detour subgraph of $Q_{n}$ is also a local detour subgraph, so $f_{l}(n) \leq f(n)$ for each $n$.

In [1], the following theorems were shown, among others:

Theorem A. For each positive integer $n, f(n) /\left|E\left(Q_{n}\right)\right|<3 / \sqrt{2 n}$.

Theorem B. For each positive integer $n, f_{l}(n) /\left|E\left(Q_{n}\right)\right|<\sqrt{3 / 2 n}$.

Theorem C. If $G$ is a local detour subgraph of $Q_{n}$, then $|E(G)| \geq 2 \cdot 2^{n}(1-$ $o(1))$. In other words, $f_{l}(n) \geq 2 \cdot 2^{n}(1-o(1))$.

Theorem D. Each local detour subgraph of $Q_{n}$ contains a vertex of degree at least $\sqrt{n}$. In other words, $F_{l}(n) \geq \sqrt{n}$.

Also Erdös et al. [1] asked the following question.

Question. Is $f_{l}(n)=f$ ?

In this article, we improve Theorems $\mathrm{B}$ and $\mathrm{C}$ by finding the asymptotics of $f_{l}(n)$ and show that $f_{l}(n)<f(n)$. We also improve Theorem $\mathrm{D}$ and find a matching upper bound, which gives the right order of magnitude of $F_{l}(n)$.

Namely, in the next section we construct a local detour subgraph of $Q_{n}$ with $3 \cdot 2^{n}(1+o(1))$ edges and a local detour subgraph of $Q_{n}$ with maximum degree at most $1.5 \sqrt{2 n}$. In the last section, we prove that $(i)$ any local detour subgraph has 
a vertex of degree $\sqrt{2 n}-0.5$ or more, (ii) any local detour subgraph of $Q_{n}$ has at least $3 \cdot 2^{n}(1-\sqrt{112 / n})$ edges, and (iii) any subgraph of $Q_{n}$ with the 2-detour property has at least $(3.000013-o(1)) \cdot 2^{n}$ edges.

This answers the Question in the negative.

\section{CONSTRUCTIONS}

In this section, we establish upper bounds for $f_{l}(n)$ and $F_{l}(n)$. To do that we need the following result by Kabatianski and Panchenko [2].

Lemma 1 [2]. $\quad$ The m-dimensional hypercube $Q_{m}$ has a dominating set $D \subset$ $V\left(Q_{m}\right)$ with $|D|=(1+o(1)) 2^{m} / m$.

Theorem 1. For every $n$ there is a subgraph $G$ of $Q_{n}$ with the 1-detour property, which has $3 \cdot 2^{n}(1+o(1))$ edges.

Proof. In this proof we write vertices of $Q_{j}$ as binary $j$-tuples. First, we define two subsets $S_{1}$ and $S_{2}$ of $V\left(Q_{n}\right)$. Let $m=\lfloor n / 2\rfloor$. Represent $Q_{n}$ as $Q_{m} \times Q_{n-m}$, where the $Q_{m}$ is induced by the first $m$ coordinates and the $Q_{n-m}$ by the last $n-m$ coordinates of elements of our $Q_{n}$. Each vector $w \in Q_{n}$ can be written as $w=v u$, where $v \in V\left(Q_{m}\right)$ is an $m$-tuple, and $u \in V\left(Q_{n-m}\right)$ is an $(n-m)$-tuple. By Lemma 1 , the $m$-dimensional hypercube $Q_{m}$ has a dominating set $D_{1}$ of size $(1+o(1)) 2^{m} / m$, and the $(n-m)$-dimensional hypercube $Q_{n-m}$ has a dominating set $D_{2}$ of size $(1+o(1)) 2^{n-m} /(n-m)$. Let

$$
\begin{gathered}
S_{1}=\left\{w=v u \in V\left(Q_{n}\right) \mid v \in D_{1}, u \in V\left(Q_{n-m}\right)\right\}, \\
S_{2}=\left\{w=v u \in V\left(Q_{n}\right) \mid v \in V\left(Q_{m}\right), u \in D_{2}\right\} .
\end{gathered}
$$

Then $\left|S_{1}\right|=2^{n-m} \cdot(1+o(1)) 2^{m} / m=(1+o(1)) 2^{n+1} / n$, and, similarly, $\left|S_{2}\right|=$ $(1+o(1)) 2^{n+1} / n$. Let $S=S_{1} \cup S_{2}$. Clearly, $|S|=(1+o(1)) 2^{n+2} / n$.

Now, let $E(G)$ consist of those edges of $Q_{n}$ that are incident with $S$, and let $G$ be the subgraph of $Q_{n}$ spanned by the edge set $E(G)$. We need to show that $G$ has the 1-detour property, and $|E(G)|=3 \cdot 2^{n} \cdot(1+o(1))$. To prove the latter

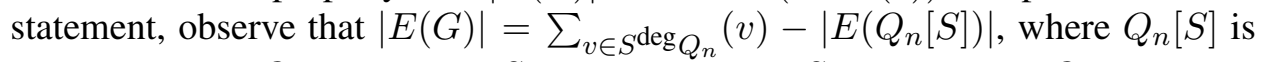
the subgraph of $Q_{n}$ induced by $S$. Since each $w \in S$ is adjacent in $Q_{n}$ to at least $m$ vertices in $S$, we get $|E(G)| \leq n|S|-0.5 m|S|=(3+o(1)) 2^{n}$.

Finally, let $w_{1}=v_{1} u_{1}$ and $w_{2}=v_{2} u_{2}$ be two vertices of $G$ that are adjacent in $Q_{n}$ but are not adjacent in $G$. Since the degree in $G$ of every vertex in $S$ is $n$, neither $w_{1}$ nor $w_{2}$ can be in $S$. The vectors $w_{1}$ and $w_{2}$ differ in exactly one coordinate, say $i$. Without loss of generality, we can presume that $i \leq m$. Thus, $w_{1}=v_{1} u$ and $w_{2}=v_{2} u$, where $v_{1}$ and $v_{2}$ differ only in coordinate $i$. By the definition, there is a vertex $x \in D_{2}$ that is adjacent to $u$. Then the vertices $y=v_{1} x$ and $z=v_{2} x$ are in $S_{2}$ and differ only in coordinate $i$. Hence, path $\left(w_{1}, y, z, w_{2}\right)$ is present in $G$. 
Remark 1. Since for $m=2^{k}-1$ the graph $Q_{m}$ has a perfect dominating set of vertices, our construction for $n=2\left(2^{k}-1\right)$ has strictly fewer than $3 \cdot 2^{n}(1-1 / n)$ edges.

Remark 2. The construction of Theorem 1 can be generalized to a $k$-detour subgraph with $\frac{1}{2}(k+1)(k+2) 2^{n}(1+o(1))$ edges; when $k$ is close to $n$; this gives little useful information, but we think that when $k$ is small relative to $n$ the result is optimal. To do so, we represent $Q_{n}$ as the direct product of $(k+1)$ smaller hypercubes of approximately equal sizes and select a minimum size dominating set in each of them. A vertex of $Q_{n}$ is included in set $S$, if its projection onto at least one of the small hypercubes belongs to its dominating set. Let graph $G$ include all edges incident with at least one vertex from $S$. Then $G$ has the $k$-detour property and $|E(G)|=\frac{1}{2}(k+1)(k+2) 2^{n}(1+o(1))$.

Theorem 2. For every $n$ there is a subgraph $G$ of $Q_{n}$ with the 1-detour property of maximum degree at most $1.5 \sqrt{2 n}-1$.

Proof. For $n<5$ the statement is trivial. Let $n \geq 5$ and $m$ be the power of 2 lying in the half-open interval $[\sqrt{n / 2}, \sqrt{2 n})$, say, $m=2^{r}$. Denote $s=\lceil(n-m+$ 1) $/ m\rceil$ and partition the set $\{1, \ldots, n\}$ into $m+1$ parts $P_{0}, \ldots, P_{m}$ so that $\left|P_{0}\right|=$ $m-1$ and $\left|P_{i}\right| \in\{s-1, s\}$ for $i=1, \cdots m$. Let $H$ be the subgraph of $Q_{n}$ spanned by the edges along the coordinates in $P_{0}$. Clearly, $H$ is the disjoint union of $2^{n-m+1}$ copies of $Q_{m-1}$. Since $m-1=2^{r}-1$, we can partition $V\left(Q_{m-1}\right)$ into $m$ perfect dominating sets $S_{1}, \ldots, S_{m}$. For $i=1, \ldots, m$, let $S_{i}^{\prime}$ denote the union of translates of $S_{i}$ over all copies of $Q_{m-1}$ in $H$. Then every $S_{i}^{\prime}$ is a dominating set in $H$ and induces in $Q_{n}$ the disjoint union of $2^{m-1} / m$ copies of $Q_{n-m+1}$. Now, for $i=1, \ldots, m$, add to $H$ the edges along the coordinates in $P_{i}$ that are adjacent to the vertices in $S_{i}^{\prime}$. This is the desired graph $G$.

In order to see that $G$ is a local detour subgraph of $Q_{n}$, consider an arbitrary edge $(u, v) \in E\left(Q_{n}\right) \backslash E(G)$. The vertices $u$ and $v$ differ in some coordinate $i \in$ $\{1, \ldots, n\} \backslash P_{0}$, say, $i \in P_{1}$. That means that they belong to different components, say $C_{u}$ and $C_{v}$, of $H$, which are adjacent in $Q_{n}$. Then the common projection of $u$ and $v$ into a copy of $Q_{m-1}$ has a neighbor $z$ in $S_{1}$. Let $z_{u}$ and $z_{v}$ be the images of $z$ in $C_{u}$ and $C_{v}$, respectively. By the definition, $z_{u}$ and $z_{v}$ are adjacent. Then $\left(u, z_{u}, z_{v}, v\right)$ is a path in $G$.

The maximum degree in $G$ is

$$
m-1+s \leq m-1+n / m \leq \sqrt{n / 2}+\sqrt{2 n}-1=1.5 \sqrt{2 n}-1 .
$$

Theorem 2 together with Theorem 3 below gives the order of magnitude of $F_{l}(n)$.

Remark 3. For each fixed $k$, the construction of Theorem 2 can be generalized to a $k$-detour subgraph with the maximum degree of order $n^{k /(k+1)}$. To do so, we choose $m$ as a power of 2 , which is a bit more than $n^{k /(k+1)}$, choose s just large enough so that if we partition $\{m, m+1, \ldots, n\}$ into parts of size close to $s$, say $j$ parts $P_{1}, \ldots, P_{j}$, then $\left(\begin{array}{l}j \\ k\end{array}\right) \leq m$. Then we assign to each $k$-element subset of 
$\left\{P_{1}, \ldots, P_{j}\right\}$ one of the perfect dominating sets $S_{i}$ of $Q_{m-1}$ and can argue as in the proof of the theorem.

Remark 4. It follows from the proof of Theorem 11 in [1] that any subgraph of $Q_{n}$ with the $k$-detour property has a vertex of degree of order $n^{k /(k+1)}$. This together with Remark 3 gives for each fixed $k$ the order of magnitude of the minimum possible maximum degree in the subgraphs of $Q_{n}$ with the $k$-detour property.

\section{LOWER BOUNDS}

First, we are going to improve the lower bound of Theorem D.

Theorem 3. $\quad F_{l}(n) \geq \sqrt{2 n+\frac{1}{4}}-\frac{1}{2}$.

Proof. Let $G$ be a local detour subgraph of $Q_{n}$ with maximum degree $F=$ $F_{l}(n)$. For a vertex $v$, let $d(v)$ denote the degree of $v$ in $G$. Then the total number of 2-edge paths in $G$ is exactly $\sum_{v \in V(G)} \frac{1}{2} d(v)(d(v)-1)$. A detour in $G$ is a 3-edge path, whose edges are coplanar and the end-vertices are not adjacent in $G$. There exists a detour for every edge of $Q_{n}$ missed in $G$; thus the total number of detours is at least $n 2^{n-1}-|E(G)|$. Every detour contains two 2-edge paths, and any 2-edge path in $G$ can belong to at most one detour. Thus, the total number of 2-edge paths in $G$ is at least $n 2^{n}-2|E(G)|=n 2^{n}-\sum_{v \in V(G)} d(v)$, so we have

$$
n 2^{n} \leq \sum_{v \in V(G)} \frac{1}{2} d(v)(d(v)+1) \leq \frac{1}{2} 2^{n} F(F+1),
$$

which implies $\left(F+\frac{1}{2}\right)^{2} \geq 2 n+\frac{1}{4}$.

In the rest of this section, we show that the construction of Theorem 1 is asymptotically optimal, and that for large $n$ any subgraph of the hypercube $Q_{n}$ with the 2-detour property has more edges than this construction. To this end, it is enough to consider the subgraphs $G$ of $Q_{n}$ such that

$$
|E(G)| \leq 4 \cdot 2^{n}
$$

Lemma 2. The number of vertices of degree 1 in a subgraph $G$ of $Q_{n}$ with the 1-detour property satisfying (1) is at most $8 \cdot 2^{n} / n$.

Proof. Assume indirectly that this number is greater than $8 \cdot 2^{n} / n$. As was observed in the proof of Theorem 12 of [1], the neighbor of any vertex of degree 1 in $G$ has degree $n$ and is not adjacent to any other vertex of degree 1. It follows that the number of vertices of degree $n$ in $G$ also is larger than $8 \cdot 2^{n} / n$. Therefore, $|E(G)|>0.5 n \cdot\left(8 \cdot 2^{n} / n\right)=4 \cdot 2^{n}$, a contradiction to (1).

From now on, we assume the following notation. For a subgraph $G$ of $Q_{n}$ and an arbitrary number $\alpha$, let $L=L(G, \alpha)=\left\{v \in V\left(Q^{n}\right) \mid d_{G}(v) \leq \alpha\right\}$, and $H=H(G, \alpha)=\left\{v \in V\left(Q^{n}\right) \mid d_{G}(v)>\alpha\right\}=V\left(Q^{n}\right) \backslash L$. For a vertex $v$, let $d_{L}(v)$ be the number of edges of $G$ incident with $v$ having the second end in $L$, and $d_{H}(v)$ be the number of edges incident with $v$ having the second end in $H$. 
Lemma 3. Let $G$ be a subgraph of $Q_{n}$ with the 1-detour property. Then

$$
n|L| \leq \alpha 2^{n}+2 \alpha|E(G)|+\sum_{v \in H} d_{L}(v) d_{H}(v) .
$$

Proof. Let $d(v)=d_{L}(v)+d_{H}(v)$ denote the degree of $v$ in $G$. We are going to count oriented 3-paths $\left(v_{0}, v_{1}, v_{2}, v_{3}\right)$ in $G$, where $v_{0} \in L$ and $\left\{v_{0}, v_{3}\right\} \in E\left(Q_{n}\right)$. Since any missing edge has such a detour, the number of paths with $v_{0}=v$ as their starting vertex is at least $n-d(v)$. On the other hand, the number of oriented 3 -paths where $v_{1}$ or $v_{2}$ belongs to $L$ does not exceed $2 \alpha|E(G)|$. Indeed, there are at most $2|E(G)|$ choices for the edge $\left\{v_{1}, v_{2}\right\}$. If one of its ends belongs to $L$, there are at most $\alpha$ choices for the other two edges of the path (they have to be parallel in $Q_{n}$ ). The number of oriented 3-paths in which $v_{0} \in L$ while $v_{1}, v_{2} \in H$, is at most $\sum_{v \in H} d_{L}(v) d_{H}(v)$. Indeed, let $v_{1}=v$; there are $d_{L}(v)$ choices for $v_{0}$ and $d_{H}(v)$ choices for $v_{2}$. The last vertex, $v_{3}$, is determined uniquely, because the two ending edges have to be parallel. Therefore,

$$
\sum_{v \in L}(n-d(v)) \leq 2 \alpha|E(G)|+\sum_{v \in H} d_{L}(v) d_{H}(v),
$$

which implies (2).

Theorem 4. Any subgraph of $Q_{n}$ with the 1-detour property has at least $3 \cdot 2^{n}(1-\sqrt{112 / n})$ edges.

Proof. Set $\alpha=\alpha(n)=\sqrt{\frac{9}{7} n}$. Since

$$
d_{L}(v) d_{H}(v) \leq \frac{1}{4}\left(d_{L}(v)+d_{H}(v)\right)^{2}=\frac{1}{4} d(v)^{2} \leq \frac{n}{4} d(v),
$$

Lemma 3 yields

$$
\sum_{v \in H} d(v) \geq 4|L|-\frac{4 \alpha}{n} 2^{n}-\frac{8 \alpha}{n}|E(G)| .
$$

By Lemma 2,

$$
\sum_{v \in L} d(v) \geq 2|L|-8 \cdot 2^{n} / n
$$

and, thus,

$$
2|E(G)|=\sum_{v \in V\left(Q_{n}\right)} d(v) \geq 6|L|-\frac{4 \alpha}{n} 2^{n}-\frac{8 \alpha}{n}|E(G)|-8 \cdot 2^{n} / n .
$$

Because $2|E(G)| \geq \alpha|H|$, we get

$$
2|E(G)|\left(1+\frac{6}{\alpha}+\frac{4 \alpha}{n}\right) \geq 6|H|+6|L|-\frac{4 \alpha}{n} 2^{n}-8 \cdot 2^{n} / n .
$$


It is easily verified that

$$
\left(1-\frac{2 \alpha}{3 n}-\frac{4}{3 n}\right) /\left(1+\frac{6}{\alpha}+\frac{4 \alpha}{n}\right) \geq 1-\frac{4 \alpha}{6 n}-\frac{4 \alpha}{n}-\frac{6}{\alpha}
$$

Since $|H|+|L|=2^{n}$, we finally get

$$
|E(G)| \geq 3 \cdot 2^{n}\left(1-\frac{4 \alpha}{6 n}-\frac{4 \alpha}{n}-\frac{6}{\alpha}\right)=3 \cdot 2^{n}(1-\sqrt{112 / n}) .
$$

Theorem 5. Any subgraph of $Q_{n}$ with the 2-detour property has at least $(3.000013-o(1)) \cdot 2^{n}$ edges.

Proof. We break the proof of Theorem 5 into a series of observations and lemmas. Let $S$ be a subset of $V\left(Q_{n}\right)$. We say that a pair of vertices $\left\{v^{\prime}, v^{\prime \prime}\right\}$ is an S-bridge if:

(1) $v^{\prime}, v^{\prime \prime} \in S$, and

(2) $v^{\prime}, v^{\prime \prime}$ are at a distance of 2 in both $Q_{n}$ and $G$, and

(3) The middle vertex of every 2-path from $v^{\prime}$ to $v^{\prime \prime}$ in $G$ also belongs to $S$.

Notice that any pair of vertices at a distance of 2 in $G$ is a $V\left(Q_{n}\right)$-bridge. if:

We say that an ordered quadruple of vertices $\left(v_{0}, v_{1}, v_{3}, v_{4}\right)$ in $G$ is an $S$-detour

(1) $v_{0}, v_{4}$ are at a distance of 2 in $Q_{n}$, and

(2) $\left\{v_{1}, v_{3}\right\}$ is an $S$-bridge, and

(3) $\left\{v_{0}, v_{1}\right\}$ and $\left\{v_{3}, v_{4}\right\}$ are edges of $G$ and are parallel in $Q_{n}$.

Every $S$-detour induces either one or two oriented 4-paths $\left(v_{0}, v_{1}, v_{2}, v_{3}, v_{4}\right)$ in $G$, where $v_{2} \in S$ is called a middle vertex of the detour. Thus, every $S$-detour has either one or two middle vertices.

We name a quadruple of vertices of $Q_{n}$ an empty square in $G$, if they induce a $Q_{2}$-subgraph in $Q_{n}$ and induce no edges in $G$.

We will use the notation of Lemma 3, where we set $\alpha=n^{1 / 3}$. We also denote $d(v)=d_{L}(v)+d_{H}(v)$ the degree of $v$ in $G$.

Lemma 4. The number of empty squares in $G$, where all four vertices belong to $L$, is at least $\left(\begin{array}{c}n \\ 2\end{array}\right) 2^{n-2}-\left(\begin{array}{c}n \\ 2\end{array}\right)|H|-(n-1)|E(G)|$.

Proof. The total number of $Q_{2}$ in $Q_{n}$ is $\left(\begin{array}{c}n \\ 2\end{array}\right) 2^{n-2}$. The number of $Q_{2}$ with at least one vertex in $H$ does not exceed $\left(\begin{array}{l}n \\ 2\end{array}\right)|H|$. The number of $Q_{2}$ that are not empty in $G$ does not exceed $(n-1)|E(G)|$, because every edge of $G$ belongs to $(n-1)$ squares in $Q_{n}$.

Lemma 5. The number of $V\left(Q_{n}\right)$-detours $\left(v_{0}, v_{1}, v_{3}, v_{4}\right)$ in $G$ such that $v_{0}, v_{4} \in L$ is at least $n(n-1) 2^{n-1}-2 n(n-1)|H|-4(n-1)|E(G)|$. 
Proof. Consider an empty square in $G$ in which all four vertices belong to $L$. There are 4 choices of an ordered pair of its opposite vertices. Let $\left(v_{0}, v_{4}\right)$ be such a pair. Since $G$ has a 2-detour property, there should exist an oriented path $\left(v_{0}, v_{1}, v_{2}, v_{3}, v_{4}\right)$ in $G$. It is easy to see that $\left(v_{0}, v_{1}, v_{3}, v_{4}\right)$ is a $V\left(Q_{n}\right)$-detour. Thus, Lemma 5 follows from Lemma 4.

Lemma 6. The number of $V\left(Q_{n}\right)$-detours in $G$ that are not $H$-detours does not exceed $3(n-1) \alpha^{2} 2^{n}$.

Proof. Let $\left(v_{0}, v_{1}, v_{3}, v_{4}\right)$ be a $V\left(Q_{n}\right)$-detour, which is not an $H$-detour. Then at least one of the following conditions is true:

(i) $v_{1} \in L$, or

(ii) $v_{3} \in L$, or

(iii) There is a middle vertex $v_{2}$ of this detour that belongs to $L$.

For each of these conditions, we claim that the number of $V\left(Q_{n}\right)$-detours that satisfies this particular condition does not exceed $(n-1) \alpha^{2} 2^{n}$. Indeed, consider detours with $v_{1} \in L$. There are at most $2^{n}$ choices for $v_{1} \in L$. Since $d\left(v_{1}\right) \leq \alpha$, there are at most $\alpha$ choices for $v_{0}$ and at most $\alpha$ choices for $v_{2}$. There are at most $(n-1)$ choices for $v_{3}$. The remaining vertex, $v_{4}$, is determined uniquely, because the edges $\left\{v_{0}, v_{1}\right\}$ and $\left\{v_{3}, v_{4}\right\}$ are parallel. Thus, $(n-1) \alpha^{2} 2^{n}$ is an upper bound of the number of such detours. Similarly, the same bound holds for the number of detours with $v_{3} \in L$. Finally, consider detours that satisfy (iii). There are at most $2^{n}$ choices for a middle vertex $v_{2} \in L$. Since $d\left(v_{2}\right) \leq \alpha$, there are at most $\alpha$ choices for $v_{1}$ and at most $\alpha$ choices for $v_{3}$. There are at most $(n-1)$ choices for $v_{0}$, and the remaining vertex, $v_{4}$, is determined uniquely, because the edges $\left\{v_{0}, v_{1}\right\}$ and $\left\{v_{3}, v_{4}\right\}$ are parallel.

Let $\epsilon$ be a positive constant that we will choose later. We set $H_{\epsilon}^{\prime}=\{v \in H$ : $\left.\left|2 d_{L}(v)-n\right| \leq \epsilon n,\left|2 d_{H}(v)-n\right| \leq \epsilon n\right\}$ and $H_{\epsilon}^{\prime \prime}=H \backslash H_{\epsilon}^{\prime}$. In particular, $v \in H_{\epsilon}^{\prime}$ implies $d(v) \geq(1-\epsilon) n$.

Lemma 7. The number of $H$-detours in $G$ that are not $H_{\epsilon}^{\prime}$-detours does not exceed

$$
3(n-1) \sum_{v \in H_{\epsilon}^{\prime \prime}} d(v)^{2}
$$

Proof. Similarly to the proof of Lemma 6, if an $H$-detour $\left(v_{0}, v_{1}, v_{3}, v_{4}\right)$ is not an $H_{\epsilon}^{\prime}$-detour, then either $v_{1} \in H_{\epsilon}^{\prime \prime}$, or $v_{3} \in H_{\epsilon}^{\prime \prime}$, or the detour possesses a middle vertex $v_{2} \in H_{\epsilon}^{\prime \prime}$. The number of detours which satisfy just one of these conditions does not exceed $(n-1) \sum_{v \in H_{\epsilon}^{\prime \prime}} d(v)^{2}$. For instance, for every choice of a middle vertex $v_{2} \in H_{\epsilon}^{\prime \prime}$, the number of choices of $\left(v_{1}, v_{3}\right)$ as its neighbors does not exceed $d\left(v_{2}\right)^{2}$. The number of choices of $v_{0}$ does not exceed $(n-1)$, and $v_{4}$ is determined uniquely.

Let $A_{4}$ be the family of $Q_{2}$-subgraphs of $Q_{n}$, where all 4 vertices belong to $H_{\epsilon}^{\prime}$ and all 4 edges appear in $G$. Let $A_{3}$ be the family of $Q_{2}$-subgraphs of $Q_{n}$, 
where all 4 vertices belong to $H_{\epsilon}^{\prime}$ and only 3 of the 4 edges appear in $G$. Notice that each $Q_{2}$ from $A_{3} \cup A_{4}$ induces exactly two unoriented $H_{\epsilon}^{\prime}$-bridges in $G$. Let $A_{2}$ be the family of $Q_{2}$-subgraphs of $Q_{n}$ that induce exactly one $H_{\epsilon}^{\prime}$-bridge in $G$. Such a subgraph contains a 2-path with both edges present in $G$ and all 3 vertices belonging to $H_{\epsilon}^{\prime}$, and at least one of the other two edges is missing from $G$.

\section{Lemma 8.}

$$
\left|A_{4}\right| \leq \frac{1}{32}(1+\epsilon)^{2} n^{2}\left|H_{\epsilon}^{\prime}\right|
$$

Proof. Indeed, $4\left|A_{4}\right| \leq \sum_{v \in H_{\epsilon}^{\prime}} \frac{1}{2} d_{H}(v)^{2} \leq\left|H_{\epsilon}^{\prime}\right| \cdot \frac{1}{2}\left(\frac{1}{2}(n+\epsilon n)\right)^{2}$.

Lemma 9. $\left|A_{3}\right| \leq \frac{1}{4} \epsilon(1+\epsilon) n^{2}\left|H_{\epsilon}^{\prime}\right|$.

Proof. Every $Q_{2}$-subgraph in $A_{3}$ has two vertices $v, u \in H_{\epsilon}^{\prime}$, where $\{v, u\} \in$ $E\left(Q_{n}\right) \backslash E(G)$. Given $\{v, u\}$, the other two vertices of this subgraph can be selected in at most $d_{H}(v) \leq \frac{1}{2}(1+\epsilon) n$ ways. Thus,

$$
2\left|A_{3}\right| \leq \sum_{v \in H_{\epsilon}^{\prime}}(n-d(v)) \cdot \frac{1}{2}(1+\epsilon) n \leq\left|H_{\epsilon}^{\prime}\right| \cdot \epsilon n \cdot \frac{1}{2}(1+\epsilon) n .
$$

Lemma 10. $\left|A_{2}\right| \leq \frac{1}{2} \epsilon(1+\epsilon) n^{2}\left|H_{\epsilon}^{\prime}\right|$.

Proof. Every $Q_{2}$-subgraph in $A_{2}$ has a vertex $v \in H_{\epsilon}^{\prime}$ and another vertex $u$ so that $\{v, u\} \in E\left(Q_{n}\right) \backslash E(G)$. Similarly to the proof of Lemma 9,

$$
\left|A_{2}\right| \leq \sum_{v \in H_{\epsilon}^{\prime}}(n-d(v)) \cdot \frac{1}{2}(1+\epsilon) n \leq\left|H_{\epsilon}^{\prime}\right| \cdot \epsilon n \cdot \frac{1}{2}(1+\epsilon) n .
$$

\section{Lemma 11.}

$$
\begin{array}{r}
\frac{1}{16}(1+\epsilon)^{2}(1+17 \epsilon) n^{3}\left|H_{\epsilon}^{\prime}\right| \geq n(n-1) 2^{n-1}-2 n(n-1)|H|-4(n-1)|E(G)| \\
-3(n-1) \alpha^{2} 2^{n}-3(n-1) \sum_{v \in H_{\epsilon}^{\prime \prime}} d(v)^{2}
\end{array}
$$

Proof. The total number of $H_{\epsilon}^{\prime}$-bridges is $2\left|A_{4}\right|+2\left|A_{3}\right|+\left|A_{2}\right|$. Because $2 d_{L}(v) \leq(1+\epsilon) n$ for any $v \in H_{\epsilon}^{\prime}$, we notice that any unoriented $H_{\epsilon}^{\prime}$-bridge supports at most $(1+\epsilon) n$ oriented $H_{\epsilon}^{\prime}$-detours with both ends in $L$. Thus, $(1+\epsilon) n$. $\left(2\left|A_{4}\right|+2\left|A_{3}\right|+\left|A_{2}\right|\right)$ is an upper bound for the number of oriented $H_{\epsilon}^{\prime}$-detours with both ends in $L$. The left-hand side of (3) is obtained by replacing $\left|A_{4}\right|,\left|A_{3}\right|$, and $\left|A_{2}\right|$ in the last expression by their upper bounds from Lemmas 8-10. The right-hand side of (3) is a lower bound of the number of oriented $H_{\epsilon}^{\prime}$-detours with both ends in $L$ derived from Lemmas 5-7. 
Lemma 12. For any vertex $v \in H_{\epsilon}^{\prime \prime}$,

$$
4 d_{L}(v) d_{H}(v) \leq n d(v)-\epsilon^{2} d(v)^{2} .
$$

Proof. Let $d=d(v), d_{\min }=\min \left\{d_{L}(v), d_{H}(v)\right\}, d_{\max }=\max \left\{d_{L}(v), d_{H}(v)\right\}$. If $\epsilon \geq 1$, then $H_{\epsilon}^{\prime \prime}$ is empty, so we may assume that $0<\epsilon<1$. Let $\theta$ be defined by $d_{\min }=(1-\theta) d / 2$; then $\theta \geq 0$.

The desired inequality is equivalent to $\epsilon^{2}-\theta^{2} \leq(n / d)-1$, so we prove this. Since $n \geq d$, the inequality is satisfied if $\theta \geq \epsilon$, so we may assume that $0 \leq \theta<\epsilon<1$.

For any real $\epsilon$, we have $\epsilon-\epsilon^{2}<\frac{1}{2}$ and then $\epsilon<1$ gives $2 \epsilon<1 /(1-\epsilon)$. From this and $\theta<\epsilon$, it follows first that $\epsilon+\theta<1 /(1-\epsilon)$, then that $\epsilon^{2}-\theta^{2}<\frac{\epsilon-\theta}{1-\epsilon}=\frac{1-\theta}{1-\epsilon}-1$. Now, if both $d_{L}(v)$ and $d_{H}(v)$ are greater than $(1-\epsilon) n / 2$, then the fact that $v \in H_{\epsilon}^{\prime \prime}$, implies $d_{\max }>(1+\epsilon) n / 2$, which results in $d=d_{\min }+d_{\max }>n$, and, therefore, $(1-\theta) d / 2=d_{\min } \leq(1-\epsilon) n / 2$, and so $(1-\theta) /(1-\epsilon) \leq n / d$, which gives the desired conclusion.

We are now ready to proceed with the proof of Theorem 5. Recall that by (1), $|E(G)| \leq 4 \cdot 2^{n}$. Because $|E(G)| \geq \frac{1}{2} \alpha|H|$, we may assume that $|H|=$ $\mathcal{O}\left(n^{-1 / 3} 2^{n}\right)$. Let $a(\epsilon)=\frac{1}{16}(1+\epsilon)^{2}(1+17 \epsilon)$. Since $\alpha=n^{1 / 3}$, Lemma 3 implies

$$
\sum_{v \in H} d_{L}(v) d_{H}(v) \geq n 2^{n}-\mathcal{O}\left(n^{2 / 3} 2^{n}\right),
$$

and Lemma 11 implies

$$
a(\epsilon) n^{2}\left|H_{\epsilon}^{\prime}\right|+3 \sum_{v \in H_{\epsilon}^{\prime \prime}} d(v)^{2} \geq \frac{1}{2} n 2^{n}-\mathcal{O}\left(n^{2 / 3} 2^{n}\right) .
$$

For $v \in H_{\epsilon}^{\prime}$, we have $d_{L}(v) d_{H}(v) \leq \frac{n}{4} d(v)$. For $v \in H_{\epsilon}^{\prime \prime}$, we may apply Lemma 12. Thus,

$$
\sum_{v \in H} d_{L}(v) d_{H}(v) \leq \frac{n}{4} \sum_{v \in H} d(v)-\frac{\epsilon^{2}}{4} \sum_{v \in H_{\epsilon}^{\prime \prime}} d(v)^{2}
$$

By combining the last two inequalities, we get

$$
\sum_{v \in H} d_{L}(v) d_{H}(v) \leq \frac{n}{4} \sum_{v \in H} d(v)-\frac{\epsilon^{2}}{24} n 2^{n}+\frac{a(\epsilon) \epsilon^{2}}{12} n^{2}\left|H_{\epsilon}^{\prime}\right|+\mathcal{O}\left(n^{2 / 3} 2^{n}\right) .
$$

We now estimate $(1-\epsilon) n\left|H_{\epsilon}^{\prime}\right| \leq \sum_{v \in H} d(v)$, and get

$$
\sum_{v \in H} d_{L}(v) d_{H}(v) \leq\left(\frac{1}{4}+\frac{a(\epsilon) \epsilon^{2}}{12(1-\epsilon)}\right) n \sum_{v \in H} d(v)-\frac{\epsilon^{2}}{24} n 2^{n}+\mathcal{O}\left(n^{2 / 3} 2^{n}\right) .
$$

Inequalities (4) and (5) yield

$$
\sum_{v \in H} d(v) \geq 4 \cdot \frac{1+\frac{\epsilon^{2}}{24}}{1+\frac{a(\epsilon) \epsilon^{2}}{3(1-\epsilon)}} \cdot 2^{n}-\mathcal{O}\left(n^{-1 / 3} 2^{n}\right)
$$


By Lemma 2,

$$
\sum_{v \in L} d(v) \geq 2|L|-8 \cdot 2^{n} / n=2 \cdot 2^{n}-\mathcal{O}\left(n^{-1 / 3} 2^{n}\right)
$$

and thus,

$|E(G)|=\frac{1}{2} \sum_{v \in L} d(v)+\frac{1}{2} \sum_{v \in H} d(v) \geq\left(1+2 \cdot \frac{1+\frac{\epsilon^{2}}{24}}{1+\frac{a(\epsilon) \epsilon^{2}}{3(1-\epsilon)}}\right) \cdot 2^{n}-\mathcal{O}\left(n^{-1 / 3} 2^{n}\right)$.

When we select $\epsilon=1 / 33$, the expression in the brackets in the last formula is equal to $3.00001306 \cdots$. This completes the proof of Theorem 5 .

\section{FINAL REMARKS}

We do not have any knowledge how $f_{l}(n) / 2^{n}$ changes as a function of $l$. Therefore, we cannot resolve the conjectures of [1] whether the order of magnitude of $f(n)$ is $\sqrt{n} 2^{n-1}$ or the function $f(n) / 2^{n}$ is unbounded.

\section{ACKNOWLEDGMENTS}

The authors thank one of the referees for the valuable suggestions that improved this article.

\section{References}

[1] P. Erdős, P. Hamburger, R. E. Pippert, and W. D. Weakley, Hypercube subgraphs with minimal detours. J. Graph Theory 23 (1996), 119-128.

[2] G. A. Kabatianski and V. I. Panchenko, Packings and coverings of the Hamming space by balls of unit radius. Problems Inform. Transmission 24 (1988), 261-272. 